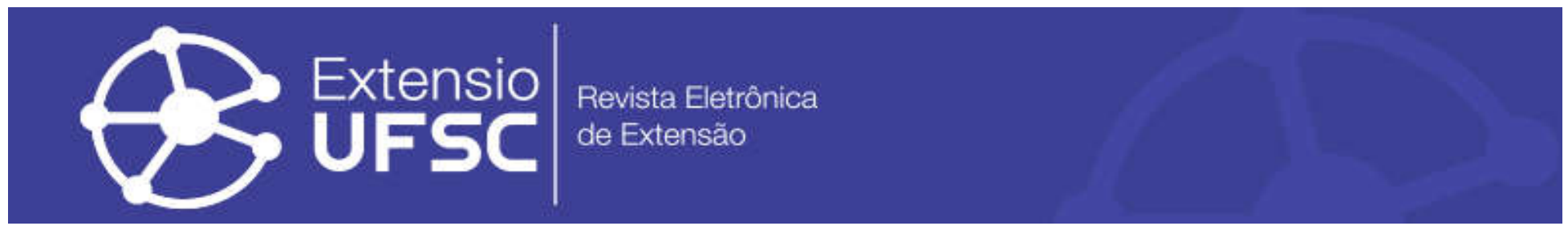

\title{
PRÁTICAS INTEGRATIVAS E COMPLEMENTARES EM SAÚDE (PICS): UM RELATO DE EXPERIÊNCIA EXTENSIONISTA
}

\author{
Alberto Sumiya \\ Universidade Federal de Santa Catarina \\ a.sumiya@ufsc.br \\ Bruna Juliana Américo Machado \\ Universidade Federal de Santa Catarina \\ bruna.julianam@gmail.com \\ Aline Ruediger Baron \\ Universidade Federal de Santa Catarina \\ aaline.baron@gmail.com
}

\author{
Sarah Pietra Rosa \\ Universidade Federal de Santa Catarina \\ sarahhpietra@gmail.com \\ Léa Beatriz Vogel Oravec \\ Universidade Federal de Santa Catarina \\ leaoravec@yahoo.com.br \\ Vanessa Mainara Marcos \\ Universidade Federal de Santa Catarina \\ vanessaemim1012@gmail.com
}

\begin{abstract}
Resumo
As Práticas Integrativas e Complementares em Saúde (PICS) no Brasil contemplam sistemas médicos complexos e recursos terapêuticos, que buscam estimular os mecanismos naturais para a recuperação da saúde. O presente estudo teve como objetivo relatar a experiência de um projeto de extensão no atendimento de estudantes universitários utilizando PICS. Houve capacitação de oito estudantes que atuaram como terapeutas aplicando auriculoterapia e meditação. Foram atendidas 64 pessoas, com média de idade geral de 24,1 $\pm 4,40$ e queixa de ansiedade (78,12\%), entre agosto e dezembro de 2019, com seis a 10 sessões. A maioria dos pacientes referiu redução das queixas. Conclui-se que as técnicas demonstraram potencial de promover saúde ao reduzir sintomas. As condições de infraestrutura de atendimento não foram adequadas necessitando-se negociação de espaço próprio.

Palavras-chaves: Terapias Complementares. Auriculoterapia. Meditação. Extensão Universitária.

\section{INTEGRATIVE AND COMPLEMENTARY PRACTICES IN HEALTH (PICS): A REPORT OF EXTENSIONIST EXPERIENCE}

\begin{abstract}
Integrative and Complementary Health Practices (PICS) in Brazil include complex medical systems and therapeutic resources, which seek to stimulate the natural mechanisms for health recovery. The present study aimed to report the experience of an extension project in the service of university students using PICS. Eight students were trained as therapists applying auriculotherapy and meditation. 64 people were attended, with an average age of $24.1 \pm 4.40$ and complaints of anxiety (78.12\%), between August and December 2019, with six to 10 sessions. Most patients reported a reduction in complaints. It is concluded that the techniques demonstrated the potential to promote health by reducing symptoms. The conditions of the service infrastructure were not adequate, requiring the negotiation of its own space.

Keywords: Complementary Therapies. Auriculotherapy. Meditation. University Extension.

\section{PRÁCTICAS DE SALUD INTEGRADORAS Y COMPLEMENTARIAS (PICS): UN INFORME DE EXPERIENCIA DE EXTENSIÓN}

\section{Resumen}

Las Prácticas de Salud Integrativas y Complementarias (PICS) en Brasil incluyen complejos sistemas médicos y recursos terapéuticos, que buscan estimular los mecanismos naturales para la recuperación de la salud. El presente estudio tuvo como objetivo reportar la experiencia de un proyecto de extensión al servicio de estudiantes universitarios utilizando PICS. Ocho estudiantes se capacitaron como terapeutas aplicando auriculoterapia y meditación. Se atendieron 64 personas, con una edad promedio de $24,1 \pm 4,40$ y quejas de ansiedad (78,12\%), entre agosto y diciembre de 2019, con seis a 10 sesiones. La mayoría de los pacientes informaron una reducción de las quejas. Se concluye que las técnicas demostraron el potencial de promover la salud al reducir los síntomas. Las condiciones de la infraestructura de servicios no eran las adecuadas, requiriendo la negociación de su propio espacio.

Palabras clave: Terapias Complementarias. Auriculoterapia. Meditación. Extensión Universitaria. 
Práticas Integrativas e Complementares em Saúde (PICS): um relato de experiência extensionista

\section{INTRODUÇÃO}

Desde o final da década de 70, a Organização Mundial de Saúde (OMS) criou o Programa de Medicina Tradicional objetivando a formulação de políticas na área. Assim, através de vários comunicados e resoluções, a OMS vem expressando o seu compromisso de incentivar seus Estados-membros a formularem e implementarem políticas públicas para o uso racional e integrado da Medicina Tradicional nos sistemas nacionais de atenção à saúde, bem como o desenvolvimento de estudos científicos com o intuito de aprimorar o conhecimento sobre sua segurança, eficácia e qualidade.

No Brasil, a legitimação e a institucionalização dessas abordagens de atenção à saúde se iniciaram a partir da década de 1980, após a criação do Sistema Único de Saúde (SUS), que trouxe a descentralização e a participação popular, levando Estados a implantarem experiências pioneiras no campo das Práticas Integrativas e Complementares em Saúde (PICS), como é chamada a Medicina Tradicional no Brasil. As PICS são definidas como recursos terapêuticos voltados à prevenção complementar de diversas doenças e promoção de saúde. As PICS podem ser tidas como parte de tratamentos paliativos, em algumas doenças crônicas, assim mostrando benefícios quando integradas a medicina convencional (BRASIL, 2018a; 2018b).

O movimento nacional brasileiro resultou na elaboração da Política Nacional de Práticas Integrativas e Complementares (PNPIC), que tem como princípios norteadores: incorporar e implementar as PICS no SUS, voltadas ao cuidado continuado, humanizado e integral em saúde; estimular as ações referentes ao controle/participação social; promover a racionalização das ações de saúde, estimulando alternativas inovadoras e socialmente contributivas ao desenvolvimento sustentável de comunidades; e contribuir com o aumento da resolubilidade do sistema de saúde e ampliação do acesso (BRASIL, 2018a; 2018b).

Atualmente, o SUS oferece gratuitamente 29 procedimentos: apiterapia, aromaterapia, arteterapia, ayurveda, biodança, bioenergética, constelação familiar, cromoterapia, dança circular e geoterapia, hipnoterapia, homeopatia, imposição das mãos, medicina antroposófica, medicina tradicional chinesa - acupuntura, meditação, musicoterapia, naturopatia, osteopatia, ozonioterapia, plantas medicinais -, fitoterapia, quiropraxia, reflexoterapia, reiki, shantala, terapia cognitiva integrativa, terapia florais, termalismo social/crenoterapia e yoga (BRASIl, 2018c).

Desta forma, as PICS podem revitalizar a assistência à saúde, amenizando o padrão biologizante e medicalizante do cuidado moderno. Por isso, torna-se essencial a fomentação da formação de profissionais para atuar nesse campo (PELECIONE, 2011), na medida em que as PICS focam os sujeitos como pessoas, não como objetos, valorizando seus contextos sociais, 
Práticas Integrativas e Complementares em Saúde (PICS): um relato de experiência extensionista

culturais e familiares, democratizando as relações terapêuticas através da dialogicidade. Esse entendimento evidencia a integralidade da saúde ao expressar novos modos de aprender e cuidar (TELESI JUNIOR, 2016).

O objetivo é relatar a experiência de um projeto de extensão no atendimento de estudantes universitários utilizando PICS.

\section{METODOLOGIA}

Relato de experiência do Projeto de Extensão denominado "Viver-Bem com PICS" da Universidade Federal de Santa Catarina (UFSC), Campus Curitibanos, no período de agosto a dezembro de 2019.

Após capacitação de oito estudantes para atuarem como terapeutas em auriculoterapia (AA) e meditação, foram convocados pacientes através de e-mail enviado à comunidade acadêmica. Posteriormente, os mesmos eram agendados para avaliação e atendimento no horário do almoço, nas dependências do Centro de Educação Profissional (CEDUP), em Curitibanos/SC.

A avaliação consistia em registrar os dados pessoais, história da queixa, patologias associadas, medicamentos, inspeção e palpação das orelhas. A aplicação de AA ocorria em salas com o paciente sentado, uma vez por semana, com duração média de 10 minutos por sessão. Como materiais eram utilizados álcool 70\%, algodão, palpadores, pinças e sementes de mostarda fixadas na orelha fita adesiva. $\mathrm{O}$ atendimento era encerrado após seis a 10 sessões com a solicitação de feedback do paciente quanto à satisfação e à percepção da melhora.

As sessões de meditação aconteciam no período vespertino, nas salas ou auditório do CEDUP, com duração de 30 minutos em média. Antecedia a meditação uma breve conversa sobre como tinham passado a semana ou se gostariam de compartilhar um pensamento/experiência ou dinâmica de grupo. Solicitava-se aos pacientes que levassem toalhas ou almofadas e a meditação se desenvolvia com ou sem música, guiada ou não, preferencialmente sentados em posição confortável, fazendo respiração nasal apenas.

O referido projeto foi registrado e aprovado no Sistema Integrado de Gerenciamento de Projetos de Pesquisa e de Extensão (SIGPEX) sob nº 201906957.

\section{RELATO DA EXPERIÊNCIA}

O projeto teve como princípio a necessidade de promover qualidade de vida e saúde aos estudantes cujas graduações são voltadas para as Ciências Rurais e Animais, sendo poucas as 
iniciativas voltadas ao cuidado físico e mental. Quando se realizou a seleção para compor o projeto, pensou-se que haveria uma rejeição, porém a procura superou as expectativas.

A capacitação dos selecionados em AA se deu em um final de semana, como costumam ser os cursos de formação. A aprendizagem da avaliação exigiu esforço, pois os graduandos eram da medicina veterinária, porém, estavam dispostos e assimilaram rapidamente o conteúdo e a prática, ganhando autonomia nos atendimentos. Os terapeutas criaram cartões de agendamento e grupo no WhatsApp ${ }^{\circledR}$ para comunicação com pacientes.

Não existia um espaço apropriado destinado ao projeto para atendimento com macas e colchonetes, por isso as salas de aula do CEDUP foram ocupadas. O local foi um fator limitante, pois dois cursos aconteciam em outro espaço, dificultando o deslocamento de algumas pessoas. Os encontros aconteciam no horário do almoço (Figura 1), para não interferir com as rotinas acadêmicas, uma vez por semana, cada terapeuta tinha agendado até quatro pacientes e a duração da sessão ficava em torno de 10 minutos, seguindo um protocolo de seis a oito sessões. Os materiais utilizados eram próprios do projeto.

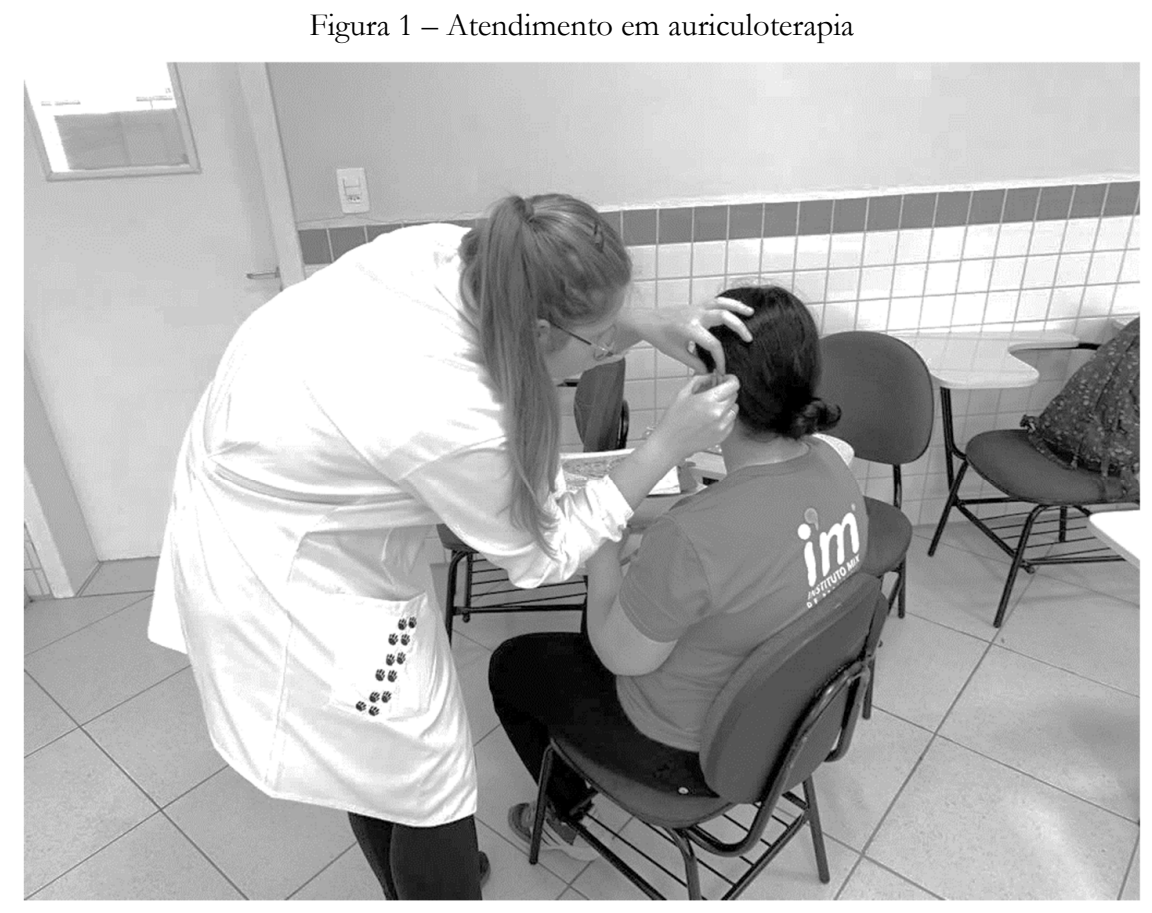

Fonte: elaboração própria

A meditação estava indicada para aqueles que tinham terminado o conjunto de sessões de AA, mas o convite esteve aberto a toda comunidade acadêmica. Contudo, houve uma adesão menor dos pacientes, por acontecer no final da tarde e eles referirem cansaço. A sessão de meditação era liderada por uma dupla de terapeutas, que para grupos novos discorria sobre os 
Práticas Integrativas e Complementares em Saúde (PICS): um relato de experiência extensionista

benefícios da meditação, a presença da prática de meditação em várias culturas, mas, no caso do projeto, salientava-se o caráter não religioso. Para grupos novos de meditação, explicava-se as posturas comuns, o tempo de duração da meditação, o uso ou não de música, tipos de respiração, a abertura ou fechamento dos olhos, utilização de almofadas e tapetes e indicação de aplicativos disponíveis em celular.

No dia da meditação, realizava-se primeiro um acolhimento onde se perguntava sobre a semana ou realizava-se uma dinâmica de grupo para os participantes interagirem, como, por exemplo, o experimento da caixa com espelho, dizendo-se haver dentro dela algo muito importante, assim, após a caixa passar por todos, solicitava-se aos presentes as reflexões ao se olhar no espelho. O tempo de meditação variou de cinco a 15 minutos, com ou sem música, guiada ou não. Sempre se aconselhava a prática diária em casa, a fim de incentivar o hábito e aumentar gradualmente o tempo.

Devido às queixas mais frequentes referidas pelos pacientes terem sido ansiedade, estresse, depressão e cansaço (Figura 2), o projeto organizou a realização de uma palestra sobre saúde mental, em formato de roda de conversa com os profissionais da assistência estudantil da UFSC/Curitibanos (Assistente Social e Psicólogo), que forneceram informações e estratégias de enfrentamento sobre as queixas que se relacionavam, de modo geral, com relações interpessoais conflituosas com professores, autocobrança e reprovações. Relataram ainda falta de ajustamento social, distância da família, problemas econômicos e a falta de atividades culturais e entretenimento em uma cidade de interior, longe de grandes centros.

Figura 2 - Sintomas autorreferidos pelos pacientes

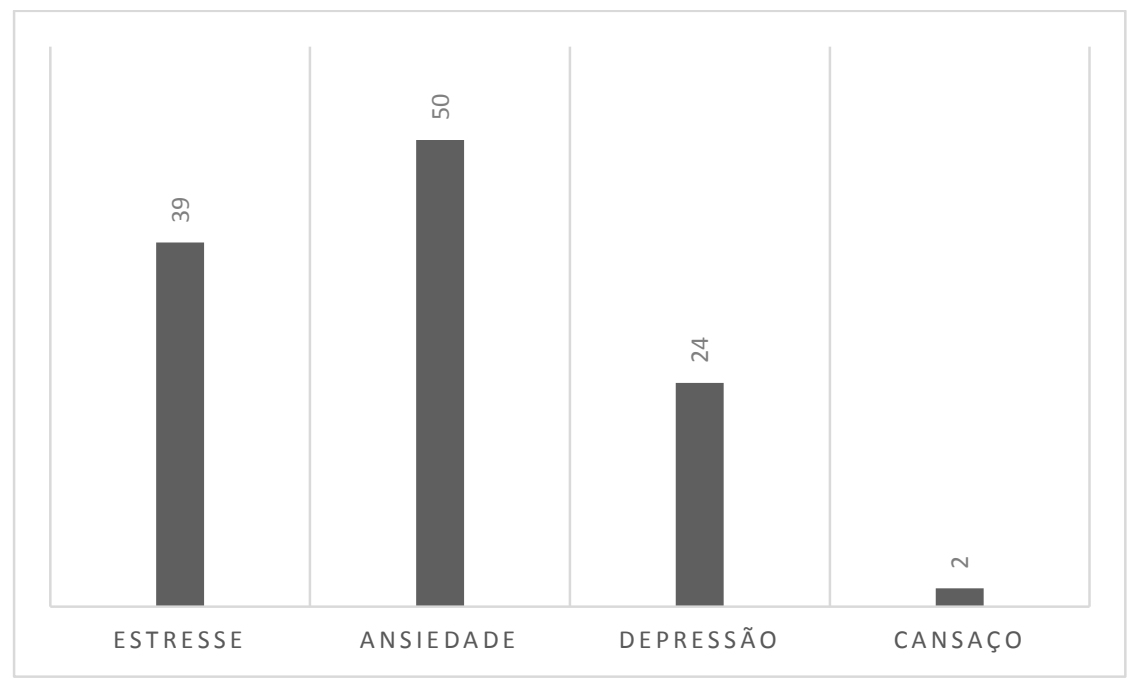

Fonte: elaboração própria 
Práticas Integrativas e Complementares em Saúde (PICS): um relato de experiência extensionista

Como relato, participaram 64 pessoas, 55 mulheres e nove homens. Destes, 58 eram estudantes de graduação, três técnicos universitários, dois estudantes de pós-graduação e um docente. A média de idade geral foi de 24,1 $\pm 4,40$ anos, e o curso com a maior participação foi o de Medicina Veterinária (76\%), seguido por Agronomia (19\%) e Engenharia Florestal (5\%). O feedback dos pacientes em relação aos cuidados recebidos foi positivo, no sentido de que referiram boa satisfação e melhora dos sintomas.

\section{DISCUSSÃO}

As PICS são uma realidade multiprofissional em saúde no Brasil, que demonstra sensibilidade, cuidado ampliado e humanizado, capacidade de integrar saberes e práticas (NASCIMENTO et al, 2018). Nesse sentido as PICS vêm representando a consolidação da promoção da saúde, na medida em que $70 \%$ da população depende dessas práticas como atenção primária à saúde devido ao aumento das doenças crônicas, aumento dos custos com os serviços de saúde, insatisfação com os serviços existentes, ressurgimento do interesse pelo cuidado holístico e preventivo, e como cuidados que oferecem qualidade de vida quando a cura não é possível. De outro modo, é uma perspectiva contra-hegemônica ao curativismo da medicina prescritiva, muitas vezes focada na doença (AMADO et al, 2017).

A experiência universitária pode ser marcada por dificuldades que podem desencadear transtornos físicos e mentais. Podem ser desafios relacionados a processos complexos e multidimensionais de transição, por exemplo, mudanças ambientais, hábitos de estudo, aquisição de novas normas de ensino-aprendizagem, implicando na criação de novos comportamentos e reflexões sobre si. Assim, o modo como cada estudante vivencia este período é único, e pode ser percebido como perda e desolação, devido à ruptura com os hábitos anteriores (POLYDORO; GUERREIRO-CASANOVA, 2010).

Proporcionar meios para a compreensão desse período é importante na redução de impactos negativos. Portanto, ao empregar diferentes procedimentos para conhecer, controlar e aprender, o estudante busca as melhores estratégias (MARTINS; ZERBINI, 2014), porém, estas podem não ser as mais adequadas à saúde e à formação, ao utilizarem estimulantes, não dormirem o suficiente, sacrificarem o lazer e os exercícios físicos, esmaecerem os laços sociais etc. Comumente, sintomas de estresse e ansiedade emergem, podendo se estabelecer como doença e solicitar acompanhamento médico e psicológico (CASTRO, 2017).

A ansiedade pode ser caracterizada como um sentimento vago e desagradável de medo, apreensão, tensão e desconforto derivados de antecipação de perigo por algo desconhecido e 
Práticas Integrativas e Complementares em Saúde (PICS): um relato de experiência extensionista

estranho, que são desproporcionais ao estímulo e qualitativamente diversos do que se observa (CASTILLO et al, 2000). Para o enfrentamento desse transtorno, o projeto relatado neste artigo ofereceu AA e meditação como recursos terapêuticos e de autocuidado.

Sabe-se que a AA atua sobre o sistema nervoso de maneira reflexa, promovendo a liberação de substâncias reguladoras e relaxantes, interferindo também com o sistema nervoso autônomo (VIEIRA et al, 2018a; ARTIOLI; TAVARES; BERTOLINI, 2019; RABISCHONG; TERAAL, 2014). Vieira et. al (2018b) mensuraram os efeitos clínicos da AA em estudantes de medicina com sintomas de ansiedade, através de um estudo randomizado, tendo como resultado menor nível de ansiedade no grupo intervenção em relação ao grupo placebo e controle.

Prado, Kurebayashi e Silva (2012) realizaram um estudo clínico randomizado, simples cego, com o objetivo de avaliar a eficácia da AA versus controle e placebo na diminuição dos níveis de estresse. Participaram 71 estudantes, que foram subdivididos em grupo controle (25), AA (24) e placebo/Sham (22). Foram realizadas de oito a 12 sessões com follow-up de 15 dias. Na análise constataram-se diferenças estatísticas significativas entre os grupos controle/auriculoterapia a partir de oito sessões, mantendo-se após a terceira e a quarta avaliação $(p=0,000)$ e entre controle/placebo $(\mathrm{p}<0,05)$, nas três avaliações. Concluiu-se que a AA obteve melhores respostas $(45,39 \%)$ do que o placebo $(34,18 \%)$.

Goldin e Gross (2010) realizaram um estudo sobre os efeitos do mindfulness (meditação focada) na ansiedade social de 14 pacientes submetidos ao exame de ressonância magnética funcional enquanto reagiam e tentavam regular crenças pessoais negativas usando como intervenções a respiração e distração focadas. Os autores concluíram que o mindfulness foi eficiente na redução de sintomas de ansiedade, depressão, experiência emocional negativa, atividade da amígdala e aumento da atividade do cérebro em regiões implicadas com o desenvolvimento da atenção e autoestima.

Mosini et al (2019) conduziram um outro estudo de revisão bibliográfica para organizar a evidência científica sobre os efeitos da meditação a partir da neurofisiologia, neuroquímica e aspectos cognitivos e comportamentais de praticantes. Encontraram 21 pesquisas e concluíram que a meditação tem efeito positivo na redução da ansiedade e estresse pela redução dos níveis de cortisol e norepinefrina, além do aumento da sensação de prazer e bem-estar pelo aumento da síntese e liberação de dopamina e serotonina.

A falta de profissionais que atuem com PICS é apontada por Habimorad et al (2020), que afirmam que a formação existente no Brasil é deficiente na graduação, pós-graduação e educação permanente, e por isso tem o potencial de disseminar conceitos errôneos e complicar a relação com a medicina convencional. Salles et al (2014) complementam os dados de Habimorad et al (2020), 
Práticas Integrativas e Complementares em Saúde (PICS): um relato de experiência extensionista

mostrando que em pesquisa com três cursos de graduação (Enfermagem, Medicina, Fisioterapia) no Brasil, constituindo uma amostra de 209 instituições públicas, somente 43 (32,3\%) ofertavam disciplinas relacionadas ao tema, predominando mais na Enfermagem $(26,4 \%)$, com carga horária média de 46 horas de maneira optativa.

Portanto, a partir da perspectiva da extensão, o projeto desenvolveu o eixo ensino-serviçocomunidade, fortalecendo a formação e o cuidado à saúde do estudante durante a experiência universitária.

\section{CONSIDERAÇÕES FINAIS}

As técnicas de auriculoterapia e meditação demonstraram descritivamente potencial de promoção de saúde, e de maneira autorreferida os pacientes com sintomas de ansiedade, estresse, depressão e cansaço informaram redução das queixas, o que refletiu na boa satisfação sobre o projeto. Apesar das técnicas não exigirem grande aparato material e de infraestrutura, as condições foram ideais, necessitando como medida a negociação de um espaço próprio para as atividades.

\section{REFERÊNCIAS}

AMADO, Daniel Miele; ROCHA, Paulo Roberto Souza; UGARTE, Olivia Albuquerque; FERRAZ, Carla Conceição, LIMA, Mélquia da Cunha, CARVALHO, Fabio Fortunato Brasil de. Política Nacional de Práticas Integrativas e Complementares no Sistema Único de Saúde 10 anos: avanços e perspectivas. Journal of Management \& Primary Health Care, v. 8, n. 2, p. 290 308, 2017.

ARTIOLI, Dérrick Patrick; TAVARES, Alana Ludemila de Freitas, BERTOLINI, Gladson Ricardo Flor. Auriculoterapia: neurofisiologia, pontos de escolha, indicações e resultados em condições musculoesqueléticas: revisão sistemática de revisões. Brazilian Journal of Pain, v. 2, n. 4, p. 356-361, 2019.

AZEVEDO, Elaine de; PELICIONI, Maria Cecília Focesi. Práticas integrativas e complementares de desafios para a educação. Trabalho, Educação e Saúde, v. 9, n. 3, p. 361-378, 2011.

BRASIL. Ministério da Saúde. Secretaria de Atenção à Saúde. Departamento de Atenção Básica. Política Nacional de Práticas Integrativas e Complementares no SUS. $2^{\mathrm{a}}$ Ed. Brasília: Ministério da Saúde. 2018a.

BRASIL. Ministério da Saúde. Secretaria de Atenção à Saúde. Departamento de Atenção Básica. Manual de implantação de serviços de práticas integrativas e complementares no SUS. Brasília: Ministério da Saúde. 2018b. 
Práticas Integrativas e Complementares em Saúde (PICS): um relato de experiência extensionista

BRASIL. Ministério da Saúde. Secretaria-Executiva. Secretaria de Atenção à Saúde. Glossário temático: práticas integrativas e complementares em saúde. Brasília: Ministério da Saúde. 2018 c.

CASTILLO, Ana Regina G. L; et al. Transtornos de ansiedade. Revista Brasileira de Psiquiatria, v. 22, n. sII, p. 20-3, 2000.

CASTRO, Vinícius Rennó. Reflexões sobre a saúde mental do estudante universitário: estudo empírico com estudantes de uma instituição pública de ensino superior. Revista Gestão em Foco, n. 9, p. 380-401, 2017.

GOLDIN, Philippe R; GROSS, James J. Effects of mindfulness-based stress reduction (MBSR) on emotion regulation in social anxiety disorder. Emotion, v. 10, n. 1, p. 83-91, 2010.

HABIMORAD, Pedro Henrique Leonetti; CATARUCCI, Fernanda Martin; BRUNO Vânia Hercília Talarico; SILVA, Ivan Beteto da; FERNANDES, Violeta Campolina; DEMARZO, Marcelo Marcos Piva; SPAGNUOLO, Regina Stella; PATRICIO, Karina Pavão. Potencialidades e fragilidades de implantação da Política Nacional de Práticas Integrativas e Complementares. Ciência \& Saúde Coletiva, v. 25, n. 2, p. 395-405, 2020

MARTINS, Lara Barros; ZERBINI, Thaís Escala de estratégias de aprendizagem: evidências de validade em contexto universitário híbrido. PsicoUSF, v. 19, n. 2, p. 317-328, 2014.

MOSINI, Amanda Cristina; SAAD, Marcelo, BRAGHETTA, Camilla Casaletti; MEDEIROS, Roberta de; PERES, Mario Fernando Prieto; LEÃO, Frederico Camelo. Neurophysiological, cognitive-behavioral and neurochemical effects in practitioners of transcendental meditation: a literature review. Revista da Associação Médica Brasileira, v. 65, n. 5, p. 706-713, 2019.

NASCIMENTO, Marilene Cabral do; ROMANO, Valéria Ferreira; CHAZAN, Ana Claudia Santos; QUARESMA, Carla Holandino. Formação em práticas integrativas e complementares em saúde. Trabalho, Educação e Saúde, v. 16, n. 2, p. 751-772, 2018.

POLYDORO, Soely; GUERREIRO-CASANOVA, Daniela. Escala de autoeficácia na formação superior: construção e estudo de validade. Avaliação Psicológica, v. 9. n. 2, p. 267-278, 2010.

PRADO, Juliana Miyuki do; KUREBAYASHI, Leonice Fumiko Sato; SILVA, Maria Júlia Paes da. Eficácia da auriculoterapia para diminuição de estresse em estudantes de enfermagem: ensaio clínico randomizado. Revista Latino-Americana de Enfermagem, v. 20, n. 4, p. 1-9, 2012.

RABISCHONG, Pierre; TERAAL, Claudie. Scientific basics of auriculotherapy: state of the art. Medical Acupuncture, v. 26, n. 2, p. 84-96, 2014.

SALLES, Léia Fortes; BEL HOMO, Rafael Fernandes; SILVA, Maria Júlia Paes da. The situation of the teaching of holistic and complementary practices in undergraduate courses in Nursing, Physiotherapy and Medicine. Cogitare Enfermagem, v. 19, n.4, p. 682-7, 2014.

TELESI JUNIOR, Emílio. Práticas integrativas e complementares em saúde, uma nova eficácia para o SUS. Estudos Avançados, v. 30, n. 86, p. 99-112, 2016. 
Práticas Integrativas e Complementares em Saúde (PICS): um relato de experiência extensionista

TESSER, Charles Dalcanale; SOUZA, Islândia Maria Carvalho de. Atenção Primária, Atenção Psicossocial, Práticas Integrativas e Complementares e suas Afinidades Eletivas. Saúde e Sociedade, v. 21, n. 2, p. 336-350, 2012.

VIEIRA, Andreia; REIS, Ana Mafalda; MATOS Luís Carlos; MACHADO, Jorge; MOREIRA, António. Does auriculotherapy have therapeutic effectiveness? Na overview of systematic reviews. Complementary Therapies in Clinical Practice, v. 33, p. 61-70, 2018.

VIEIRA, Andreia; HINZMANn, Mariana; SILVA, Karine; SANTOS, Marias João; MACHADO, Jorge. Clinical effect of auricular acupuncture in anxiety levels of students prior to the exams: A randomized controlled trial. European Journal of Integrative Medicine, v. 20, p. 188-192, 2018b.

Recebido em: 23/09/2020

Aceito em: 15/04/2021 